\title{
Educación bilingüe de frontera y políticas lingüísticas en Uruguay
}

Claudia Brovetto*

Resumen: El Uruguay no es una sociedad lingüísticamente homogénea hablante de español. El bilingüismo español-portugués que se consigna en una amplia zona del noreste de Uruguay es un ejemplo de esta realidad. Recientemente, y de un modo bastante innovador para la tradición de las políticas lingüísticas en el Uruguay (o para la falta de ellas), esta realidad sociolingüística ha sido tomada en cuenta con programas educativos específicos. Este artículo presenta un análisis de estos programas, sus antecedentes, objetivos y resultados preliminares, así como un análisis primario de dos documentos que constituyen actos de políticas lingüísticas en el Uruguay: la nueva Ley de Educación y el nuevo Programa de Educación Inicial y Primaria.

Palabras clave: educación bilingüe, inmersión, educación primaria, políticas lingüísticas

\section{Bilingual education at the Uruguayan-Brazilian border and language policies in Uruguay}

\begin{abstract}
Uruguayan society is not linguistically homogeneous in that not all its population speaks Spanish. The bilingualism present in the northeast of Uruguay is an example of this reality. Recently, in a quite innovative way for the Uruguayan traditions in language policies (or for the lack of them), this sociolinguistic reality has been considered through the implementation of specific educational programs. This paper presents an analysis of these programs, their antecedents, goals and preliminary results. It also presents an initial analysis of two documents that are events in Uruguayan language policies: the recently approved Law of Education and the new official Curriculum for Primary Schools.
\end{abstract}

Key words: bilingual education; immersion; primary school; language policies.

\section{Introducción}

Este trabajo presenta una reseña de la educación fronteriza analizada desde la cuestión lingüística, con especial detenimiento en los programas de educación bilingüe que se desarrollan desde 2003 en escuelas uruguayas de frontera. El

* Programa de Políticas Lingüísticas, Administración Nacional de Educación Pública. Montevideo, Uruguay. claudia.brovetto@gmail.com 
objetivo de este trabajo es analizar la historia y el presente de la educación fronteriza en relación a la reciente aprobación de documentos de alcance nacional con carácter de actos de políticas lingüístico-educativas.

Uruguay atraviesa un período fermental en materia de "legislación" educativa (nueva ley de educación, nueva formulación de políticas lingüísticas, nuevo programa de Educación Primaria), donde el lenguaje aparece en un lugar explícito y relevante como probablemente no haya estado en la historia de la educación del Uruguay desde sus orígenes como Estado independiente. En el contexto de esta atención particular al lenguaje en la educación, la cuestión fronteriza ocupa, a su vez, un lugar destacado.

Tradicionalmente considerado como "el problema fronterizo", la educación en la zona noreste de Uruguay ha constituido un espacio particular de interacción entre lo educativo y lo lingüístico en sus distintas dimensiones, así como entre la visión académica y la gestión de programas en el área de la educación. En este artículo se presenta un panorama de dicha compleja interacción. Comenzando por una presentación sucinta de la composición histórico-lingüística de la frontera recogida en trabajos académicos, se presentan a continuación las principales intervenciones del sistema educativo en la región, que constituyeron antecedentes y condiciones de posibilidad para los actuales programas. Luego se describen los programas de lengua vigentes actualmente y algunos estudios preliminares de su impacto en resultados de aprendizajes. Por último, se analizan brevemente dos documentos-actos de políticas lingüísticoeducativas: la Ley General de Educación (MEC, 2008) y el Programa de Educación Inicial y Primaria (CEP, 2009).

\section{Uruguay heterogéneo}

Como se ha expresado en diversos trabajos (Behares, 1984; Barrios et al., 1993; Behares, 2007; ANEP, 2008), a pesar de una larga tradición ideológicamente orientada hacia un "Uruguay cultural y lingüísticamente homogéneo", una ya importante tradición de estudios científicos muestra que el Uruguay no es una sociedad lingüísticamente homogénea en lo que respecta a la lengua mayoritaria en su territorio, el español, así como tampoco es una sociedad estrictamente monolingüe. Esta heterogeneidad tiene una doble fuente: por un lado, la existencia de grupos humanos de nacionalidad uruguaya que hablan otras lenguas distintas al español, y por otro, la diversidad dentro del español del Uruguay.

En cuanto a la primera fuente de heterogeneidad, es posible afirmar que, tomado como un todo, el Uruguay no es un país monolingüe. Lo es ciertamente una región amplia del territorio nacional; sin embargo, debido a su constitución 
histórica ciertas áreas geográficas no lo son, y no lo han sido desde hace siglos. El caso más evidente de situación de bilingüismo en el territorio nacional es el de los departamentos del norte del país, que en épocas anteriores fueron regiones de habla casi exclusivamente portuguesa, y en la actualidad zonas bilingües español-portugués (hoy reducidas a los departamentos de Rivera, Artigas y Cerro Largo). Aunque numéricamente menos importantes, subsisten en el país grupos humanos que conservan lenguas europeas traídas por los inmigrantes, como el ruso en la región de San Javier, una variedad del francés de origen valdense en Colonia o dialectos del alemán en zonas fronterizas litoraleñas con Argentina, por citar algunos ejemplos.

Además de las realidades señaladas antes, referidas a grupos con diferentes lenguas orales en calidad de lengua materna, existe en Uruguay la comunidad sorda hablante de la Lengua de Señas Uruguaya, lengua natural e histórica de estas comunidades. Esta comunidad, extensa en su dimensión, aunque difícil de precisar en cantidad de hablantes, se encuentra a lo largo del país, aunque con mayor concentración en el área metropolitana.

La segunda dimensión de heterogeneidad lingüística, tal vez menos evidente desde ámbitos no especializados, es la que hace a las diferentes variedades del español, claramente identificables en el Uruguay. Puede afirmarse que la expresión "español del Uruguay" apunta a una entidad heterogénea, ya que, como toda lengua natural, puede describirse como una compleja trama de haces de variación, especialmente en la dimensión geográfica, la social vinculada a diversas formas de estratificación, o a la generacional, para tomar solamente tres de las dimensiones de variación más evidentes ${ }^{1}$ (Elizaincín, 1992 y 1995).

\section{El Uruguay fronterizo. Historia y presente}

La zona fronteriza del norte del país se caracteriza, en lo que tiene que ver con la situación lingüística, por la presencia de dos lenguas, el español y el portugués, razón por la cual es una región bilingüe. Además de la presencia del español y el "portugués brasileño", en esta región se hablan otras variedades lingüísticas que han sido denominadas informalmente "portuñol", "fronterizo", "bayano" o "brasilero". Una importante tradición científica de estudios fronterizos realizados en forma continua desde la década de 1950, nos permite afirmar

1. En lo que respecta a lo espacial o geográfico, la investigación ha podido establecer la existencia de tres áreas lingüísticas diferenciadas: a) Un español "rioplatense", muy semejante en sus rasgos característicos al español hablado en la provincia de Buenos Aires, por ejemplo, aunque con rasgos variacionales propios, b) Un español "litoraleño", cercano en sus características al hablado en las provincias argentinas del litoral del Río Uruguay y c) Un español del "nordeste", desarrollado en contacto con el Portugués del Uruguay, y sensiblemente diferente en sus características con respecto a los otros dos. 
que estas variedades dialectales tienen base portuguesa, es decir que son, desde el punto de vista lingüístico, variedades del portugués, aunque ciertamente influidas por el contacto con el español. Estas variedades lingüísticas fronterizas han sido denominadas en trabajos científicos DPU (Dialectos Portugueses del Uruguay) y más recientemente portugués del Uruguay. El portugués del Uruguay es, como todas las lenguas, una estructura compleja y sistemática, cuya gramática puede ser descrita y analizada en sus diversos niveles (ver Behares, 2007 y Carvalho, 2007). Es también una variedad lingüística ágrafa, razón por la cual tiene menos estabilidad y fijación que las variedades con larga tradición escrita como el español o el portugués estándar. Carvalho (2007) asigna al portugués del Uruguay dos características principales: su calidad de variedad rural (rasgos lingüísticos del portugués "gaúcho" rural) y de contacto (influencia del español debido a dos siglos de contacto lingüístico).

La sociedad fronteriza es, por lo tanto, una sociedad bilingüe con presencia del español y del portugués del Uruguay. La presencia de la lengua portuguesa en territorio uruguayo se debe a razones históricas que se remontan a los $S$. XVII y XVIII, en los cuales estas áreas geográficas comienzan a poblarse y a oscilar en su posesión por las coronas española y portuguesa, así como por las Misiones Jesuíticas. La historia de la región fronteriza indica entonces que la presencia del portugués en esta zona no se explica por un "avance" de esta lengua desde Brasil, como muchas veces se piensa, sino que es lo que se denomina una lengua "de herencia".

Considerando el diferente estatus social del español y el portugués y sus ámbitos de uso diferenciados, esta sociedad ha sido caracterizada además como diglósica, con el español como variedad alta y el portugués del Uruguay como variedad baja. Como es característico en las sociedades con bilingüismo y diglosia, el portugués es la lengua que se usa en los ámbitos familiares, íntimos y coloquiales, mientras que el español cumple las funciones de lengua oficial del país, es usada en los ámbitos públicos, como las oficinas, las empresas, los medios de comunicación y las instituciones educativas. El uso del portugués del Uruguay es el que típicamente tienen las lenguas minoritarias que se restringen a ciertos ámbitos de funcionamiento, en el contexto de una sociedad cuya lengua mayoritaria es el español ${ }^{3}$.

2. Si bien nos referimos aquí a "la Frontera" como una unidad, es necesario notar que se trata de una región heterogénea en su conformación histórica y social, lo cual dio como resultado distintos escenarios sociolingüísticos en la actualidad. En Bertolotti et al. (2005) y Coll (2009) se presentan y analizan las diferencias históricas de la zona fronteriza que explican la presencia relativa del portugués y el español en las diferentes regiones de "la Frontera".

3. La diglosia fronteriza ha sufrido diversas modificaciones a lo largo de la historia. Behares (2007) presenta una interesante y reveladora explicación de la evolución de la matriz diglósica fronteriza 
El portugués del Uruguay se encuentra además socialmente estratificado, con una mayor presencia en los sectores más humildes y menos urbanizados de la sociedad fronteriza; es una variedad lingüística estigmatizada, es decir, es una lengua sin prestigio y considerada incorrecta a veces hasta por sus propios hablantes. El español, por su parte, es la lengua de las clases medias y altas urbanizadas (Carvalho, 2007).

Por las razones expuestas, en anteriores trabajos hemos definido al portugués del Uruguay como una lengua fronteriza, minoritaria, y de herencia (Brovetto, Geymonat y Brian, 2007). De modo complementario, Carvalho (2007) lo define como una lengua rural y de contacto. Carvalho argumenta además que estos rasgos oscilan en un continuo dialectal "portugués uruguayo rural portugués uruguayo urbano - portugués brasileño urbano". En un estudio con hablantes de Rivera y Artigas, Carvalho muestra que los hablantes oscilan en la presencia de diversos rasgos y se ubican en el continuo según sus características sociales y las condiciones de interacción. De este modo, más que una dicotomía entre portugués del Uruguay (o DPU) y portugués más estándar, se observa una situación de frecuencia condicionada por variables sociales y estilísticas. (Carvalho, 2003a; 2003b y 2004).

Diversos estudios han señalado que existe un porcentaje de hablantes nativos monolingües en portugués del Uruguay, que sólo aprenden el español en la escuela. Como se explica en detalle en Brovetto, Geymonat y Brian (2007), esta compleja situación sociolingüística no ha sido considerada en la historia de la educación fronteriza. El rol tradicional de la escuela ha sido el de reforzar y promover el uso del español y corregir las "hablas incorrectas" típicas de la zona. A nivel de la práctica escolar, esto supuso una represión sistemática del uso del portugués en las escuelas, con los perjuicios académicos y afectivos que ello implica para estos niños.

La represión del uso del portugués en las escuelas constituye una actitud problemática ya que tiene consecuencias negativas a varios niveles. En primer lugar, está basada en un desconocimiento de la realidad lingüística local, esto es, de la situación social de bilingüismo. En segundo lugar, se apoya sobre la premisa de que el portugués del Uruguay es una forma "incorrecta" de comunicación, lo cual supone una ignorancia acerca de la naturaleza y el funcionamiento de las lenguas naturales, ya que desde el punto de vista estrictamente lingüístico, esta variedad del portugués es una lengua compleja y completa que sirve para las necesidades de una comunidad de usuarios. En tercer lugar, la represión del uso del portugués en las escuelas desconoce los

que explica la interacción de las lenguas y sus variedades en relación a la modificación de la estructura social y las condicionantes políticas. 
beneficios cognitivos y sociales del bilingüismo. Finalmente, y tal vez uno de los aspectos fundamentales a tener en cuenta, esta actitud desconoce la importancia social y psicológica del respeto por la lengua materna del niño (UNESCO, 1953).

\section{Hacia el establecimiento de la educación bilingüe de frontera}

En esta sección se presentan dos antecedentes fundamentales de los actuales programas de educación bilingüe español - portugués en área de frontera, en particular, el proyecto de la Prof. García Etchegoyen de Lorenzo (Lorenzo, 1967 y 1975) y el Programa Pedagógico Experimental en Lectura y Escritura de Rivera (PROPELER) (Behares y Anollés, 1990).

Como se ha señalado en varios trabajos (Elizaincín, 1979; Behares, 1985; Barrios, 1996; Colombo 1996), el Uruguay es un país con escasa planificación lingüística ${ }^{4}$. La frontera de habla portuguesa es en un ejemplo de esta carencia. De hecho, fue la escuela pública vareliana el gran mecanismo de regulación lingüística en la región. Sin embargo, se trata de un emprendimiento tardío, se puede datar la acción de la escuela estatal en español en la frontera recién a partir de la segunda década del Siglo XX.

Hasta ese entonces el país todo no había sido planificado en el plano educativo tampoco. Teniendo en cuenta la inmigración multinacional que nutrió al Uruguay entre 1860 y 1920, puede decirse que el resultado esperado habría de ser una sociedad plurilingüe. Sin embargo, no fue ese el resultado, gracias fundamentalmente al efecto de la Ley de Educación Común, que redactara José Pedro Varela y que fuera aprobada con modificaciones en el Decreto-Ley que lleva el nombre de "Reglamento de la Instrucción Pública" por el Gobierno de Latorre en 1877. Este Decreto consagraba la educación generalizada, laica, gratuita y obligatoria, y el uso de la lengua española ("Idioma Nacional") en todas las escuelas del país (Art. 38: "En todas las escuelas públicas la enseñanza se dará en el Idioma Nacional"). Al desconocer las hablas de los inmigrantes, y de sus potenciales hijos y nietos, este decreto se constituye en un primer acto de planificación lingüística.

Esta política educativo-lingüística fue seguida al pie de la letra en todo el territorio y, especialmente, en la frontera, donde los lusohablantes fueron escolarizados a partir del modelo de los hispanohablantes del sur. Como explica Behares (1985, p. 16) la educación monolingüe en el norte dio por resultado una sociedad bilingüe: "El bilingüismo fronterizo es un resultado extraordinario de la planificación lingüística”.

4. Para un análisis de los modos de establecimiento de las políticas lingüísticas ver Behares y Brovetto (2009a y 2009b). 
En términos de didáctica lingüística la fuerte impronta monolingüe se expresa mediante la enseñanza exclusiva en español, que ignora y deja suspendida la competencia lingüística materna de los hablantes mediante la prohibición expresa de utilizar el portugués. Esta estrategia de planificación lingüístico-educativa en la frontera y las prácticas escolares fronterizas se mantuvo incambiada hasta 1967 , en que se discutió por primera vez un plan de reforma. A partir del "revolucionario descubrimiento" de Rona (1959) que desvendó la existencia del "Dialecto Fronterizo", la Profa. Eloísa García Etchegoyen de Lorenzo elaboró un proyecto que presentó en el Consejo Nacional de Enseñanza Primaria y Normal para ser aplicado en la zona fronteriza (Lorenzo, 1967 y 1975). Dicho documento propone tomar algunas escuelas de Rivera, homogéneas en lo socio-geográfico, para seguir aplicando en algunas de ellas la enseñanza monolingüe en español, en otras ir incorporándolo con metodología de segunda lengua y en otras aplicar un programa de educación bilingüe español-portugués. Los resultados obtenidos en cada caso serían analizados y contrapuestos, a fin de estudiar cuál podía ser el encare pedagógico-didáctico más adecuado. Este proyecto fue finalmente rechazado, más por cuestiones ideológicas vinculadas a la soberanía nacional y el supuesto carácter amenazador del portugués, que por cuestiones técnicas (Behares, 2007).

El siguiente antecedente significativo en materia de intervención educativa sobre la cuestión lingüística fronteriza, luego de las siguientes dos décadas caracterizadas por el autoritarismo pedagógico propio de la dictadura militar, lo constituye el Programa Pedagógico Experimental en Lectura y Escritura de Rivera (PROPELER), desarrollado en 1989 y 1990 a través de un acuerdo entre la Universidad de la República y el Consejo de Enseñanza Primaria. El programa consistía en el trabajo conjunto de maestros de algunas escuelas de Rivera con un grupo de especialistas (lingüistas, psicólogos, antropólogos y pedagogos) para la elaboración de estrategias para procesar la adquisición de la lengua escrita en ese contexto. Este procedimiento apuntaba a darle la posibilidad a los maestros fronterizos de contextualizar las directivas programáticas centralistas y de corte monolingüe a la realidad particular, a través de un proceso reflexivo y de negociación constante con sus niños, los colegas y los técnicos. Aunque la lengua escrita fuera el objeto focalizado, el programa revisaba y proponía reflexionar acerca de todo el funcionamiento didáctico. En lo que respecta al portugués del Uruguay (o los DPU) la experiencia mostró la posibilidad de utilizarlo como el centro del saber lingüístico de niños y maestros y como el punto de partida e impulsor de otros aprendizajes, a saber el español y el portugués estándares (Behares y Anollés, 1990). En 1991, y sin justificación ni explicaciones explícitas, el programa fue eliminado por el nuevo Consejo de Enseñanza Primaria. 
La década de los 90, especialmente en el Departamento de Rivera, se caracterizó por notorios cambios de actitudes en los docentes y en las autoridades. Behares (2007) menciona algunos procesos que subyacen a estos cambios: a) La difusión a todos los niveles de la experiencia del PROPELER; b) La constitución del Grupo de Investigadores de Lenguaje y Educación en Áreas de Frontera en el seno de la Asociación de Universidades "Grupo Montevideo" en 1995; c) La inclusión de materiales fronterizos en los textos escolares con "Guía del Maestro", publicados entre 1999 y 2000 con financiación de ANEP y con amplia difusión (Garibaldi y Salvo, 1999 y 2000); d) La realización de una Maestría en Estudios Fronterizos en Montevideo (1999-2001) y una Diplomatura en Estudios Fronterizos en Rivera (2001-2002) por la Universidad de la República, con importante participación de maestros e inspectores de Enseñanza Primaria.

\section{Educación bilingüe de frontera en el S. XX|}

Los actuales programas de educación bilingüe de frontera surgen en los albores del S. XXI, y son, en gran medida, el resultado esperado de sus antecesores y del contexto socio-institucional presentado antes. El Programa de Inmersión Dual español - portugués comenzó en 2003 en dos escuelas y el Programa de Enseñanza de Portugués por Contenidos Curriculares, en 2006 en tres escuelas. A partir de ese momento han ido creciendo en número de escuelas de modo constante; en el año 2009 estos programas funcionan en un total de 36 escuelas y alcanzan a una población estimada de 7000 niños.

La metodología de inmersión supone la utilización del portugués para la presentación de contenidos curriculares, sin constituir el foco de atención explícita de la actividad escolar. Es decir, en principio, no se enseña la lengua a través de la presentación explícita de sus estructuras, sino que se utiliza como vehículo de enseñanza de contenidos curriculares programáticos en diversas áreas, por parte de un maestro de Educación Primaria con competencia avanzada en portugués. En este modelo metodológico, el docente utiliza solo el portugués para toda la comunicación con los niños, dentro y fuera del salón de clase. De aquí que se haga referencia a los programas de inmersión como enseñanza en portugués y no de portugués. La modalidad particular de inmersión en el caso de las escuelas bilingües fronterizas es la de "inmersión dual" (dual immersion, two-way immersion), que se caracteriza por reunir en las aulas del programa a niños hablantes de las distintas lenguas de instrucción. En las aulas de inmersión dual cada grupo trabaja alternadamente con dos maestros, uno que enseña en español y otro en portugués. 
Además del programa de inmersión dual, que supone un modelo fuerte de educación bilingüe, en 2006 se crea el Programa de Enseñanza de Portugués por Contenidos Curriculares, como una adaptación del modelo de educación bilingüe a la escuela de horario simple. A diferencia del programa de inmersión, esta modalidad de trabajo tiene un formato más de tipo "clase de lengua" en tanto se trabaja en portugués durante tres o cuatro horas semanales. Sin embargo, dada la orientación metodológica ofrecida por el equipo técnico y el trabajo en formación de docentes, así como el hecho de que las aulas de portugués están a cargo de maestros de Primaria, el programa se aleja de las modalidades tradicionales de enseñanza de la lengua, para acercarse a un escenario más característico de la educación bilingüe 5 . La variedad de portugués usada en el salón de clase es el "portugués estándar brasileño", probablemente en su norma "gauicha".

En la justificación oficial de estos programas (inmersión dual español portugués, y de enseñanza de portugués por contenidos curriculares) se hace explícita la consideración del contexto sociolingüístico local y la importancia de la inclusión del portugués en la educación en tanto estándar vinculado a la variedad vernacular. De un modo inédito en la escuela pública uruguaya, con los antecedentes mencionados como condiciones de posibilidad, estos programas se plantean los siguientes objetivos: formar individuos bilingües y bidialectales, respetar y valorizar las variedades lingüísticas fronterizas, desarrollar la oralidad y la escritura en español y portugués estándar (Brovetto, Brian, Díaz y Geymonat, 2004; Brovetto, Geymonat y Brian, 2007; Quesada y Geymonat, 2007 y Quesada y Geymonat, 2008) ${ }^{6}$.

5. El Programa de Enseñanza de Portugués por Contenidos Curriculares encierra de algún modo una contradicción existencial que no pasó desapercibida para el equipo técnico que lo implementó y que lo lleva adelante y que radica en la paradoja de proponer un programa de enseñanza de portugués a una población que ya es hablante de portugués como primera o segunda lengua, en alguna de sus variedades. Por un lado, el modelo deseado de trabajo con las lenguas en escuelas de frontera es el de educación bilingüe en portugués; por otro, las condiciones de la mayoría de las escuelas públicas, que son de horario simple, y el escaso o nulo crecimiento del modelo escolar de "Tiempo Completo" imponía la necesidad de un diseño alternativo, de menor carga horaria. A pesar de esta dificultad, el hecho mismo de la presencia del portugués en las aulas de frontera en la voz de maestros de Primaria es de por sí factor cuestionador de la impronta monolingüe "sólo español" y potencialmente transformador de las prácticas lingüísticas negadoras y represivas.

6. Debe señalarse, sin embargo, que estos objetivos, planteados en diversos documentos, publicaciones y presentaciones en conferencias por parte del equipo técnico del programa y presentes como pautas orientadoras en la capacitación de los docentes no constan en resoluciones oficiales de creación de los programas. Es sólo con el advenimiento de los Documentos de la Comisión de Políticas Lingüísticas en la Educación Pública (ANEP 2008) y el nuevo Programa de Educación Inicial y Primaria (CEP 2008) que se hacen explícitos estos objetivos. Debe anotarse también que estos argumentos referidos a la situación sociolingüística e histórica de la frontera 


\section{Acerca de la "mezcla" de lenguas y su relación con la educación bilingüe}

En esta sección se presentan los resultados de dos estudios de evaluación primaria del impacto del programa de inmersión dual en escuelas de frontera. Ambos estudios fueron realizados por el equipo técnico del programa y, aunque no revisten carácter de evaluaciones externas, constituyen miradas preliminares evaluativas del impacto de la educación bilingüe en los resultados de aprendizaje, en particular, en el desarrollo de la lengua escrita en español.

Uno de estos trabajos es un estudio longitudinal de adquisición de la lengua escrita en español de un grupo de niños de frontera (Brovetto, Geymonat y Brian, 2007). Dos grupos de ocho niños cada uno participaron en el estudio: uno de ellos asistía a escuelas de Tiempo Completo bilingües por inmersión dual y el otro a escuelas de Tiempo Completo monolingües en español, de similares características (ubicación geográfica, nivel social). Todos los niños eran hablantes nativos de portugués del Uruguay. Se tomó registro de producciones escritas en español de los 16 niños durante dos años $\left(1^{\circ}\right.$ y $2^{\circ}$ año escolar) en cuatro momentos, en similares condiciones de producción. Los trabajos escritos fueron analizadas de acuerdo a una herramienta adaptada por los autores en base a distintas fuentes que consideraban los siguientes aspectos de la producción escrita: (a) Ideas / Contenido, (b) Organización, (c) Vocabulario, (d) Estructura oracional y Fluidez, (e) Corrección gramatical, (f) Aspectos convencionales. Cada una de estas áreas era evaluada y se le asignaba un nivel. A partir de este análisis, a cada producción escrita se le asignaba un nivel global en la siguiente escala: (1) Preconvencional, (2) Emergente, (3) Inicial Bajo, (4) Inicial Alto, (5) Transicional, y (6) Competente.

El estudio mostró que las producciones escritas de los niños al final de $2^{\circ}$ año escolar se ubicaban entre el nivel "Inicial Alto" y "Transicional" y no existían diferencias significativas en el nivel de las producciones en español de los niños de ambos grupos, a pesar de que los niños en escuelas bilingües tenían solo la mitad del tiempo de exposición y trabajo en español en comparación con el grupo de niños que asistían a escuelas monolingües. Se constató además, en

siempre han estado interferidos por dos argumentos instalados como persistencias discursivas: por un lado, las justificaciones en torno a la integración regional y los compromisos de políticas lingüísticas asumidos en el marco del MERCOSUR, y por otro, los vinculados a una visión de tipo purista cuya aspiración implícita en el fuero íntimo es la enseñanza de los estándares que termine con las hablas incorrectas, es decir, la fantasía de eliminación del portugués del Uruguay. Estas persistencias discursivas tienen variado origen y atraviesan la sociedad en sus distintos niveles: autoridades de la educación en distintos períodos, docentes de frontera y de otras regiones, medios de comunicación, la comunidad escolar (ver, entre otros, Barrios, 2006 y Brovetto et al., 2007). 
promedio, una tendencia mejor (aunque estadísticamente no significativa) en las producciones de los niños en escuelas bilingües (Brovetto et al., 2007).

Un estudio siguiente sobre los mismo datos recogidos (Geymonat et al., 2007) analizó la presencia de marcas de la lengua materna (portugués del Uruguay) en las producciones escritas en español. Se analizó la presencia de interferencias léxicas (e.g. "el gato acordó", por "el gato se despertó”), y morfosintácticas (e.g. "subió en el árbol”, por "subió al árbol”). El estudio mostró que en $1^{\circ}$ año (primeras dos muestras de producciones escritas) se registran similares interferencias del portugués en ambos grupos de niños. En $2^{\circ}$ año, en cambio, luego de dos años de educación bilingüe, las producciones de los niños en escuelas bilingües muestra un número de interferencias significativamente menor que las producciones de los niños en escuelas monolingües. El trabajo concluye que las interferencias del portugués (lengua materna) en las producciones escritas en español (segunda lengua) son significativamente menos en los niños bilingües que asisten a escuelas bilingües español-portugués, que en los niños bilingües que asisten a escuelas monolingües en español. Esto sugiere que la introducción simultánea de la escritura, o la educación bilingüe en general, ayudarían a la separación temprana de los sistemas lingüísticos de los niños bilingües.

Estos resultados constituyen, aunque todavía modestamente dado su carácter preliminar y el reducido número de la muestra, un indicador de los beneficios de la educación bilingüe de frontera y un fuerte contra-argumento al discurso purista en torno a los efectos negativos de la educación bilingüe para el desarrollo del español.

\section{Nuevos actos de políticas lingüísticas: Ley de Educación y Programa de Educación Primaria}

En esta sección se trata la presencia de la cuestión fronteriza y sus peculiaridades lingüísticas en dos documentos fundamentales con alcance nacional y con carácter de actos de políticas lingüísticas: la Ley General de Educación No. 18.437 (MEC, 2008) y el Programa de Educación Inicial y Primaria (CEP, 2008). Estos dos documentos muestran, aunque con algunas diferencias derivadas de sus diversos objetivos y ámbitos de producción, un grado de interacción y articulación que resulta novedoso en la tradición de establecimiento de políticas lingüísticas en Uruguay.

En trabajos anteriores (Behares y Brovetto, 2009a y 2009b) planteamos algunas hipótesis en relación a los modos de establecimiento de las políticas lingüísticas en Uruguay, a los mecanismos y procedimientos que el Estado se ha dado para establecer intenciones en el área del uso, estatus y enseñanza de 
las lenguas (sean estas lengua materna, primera, segunda, o extranjera), sonora o gestual, oral o escrita. Estos trabajos plantean como primera hipótesis la existencia en Uruguay de tres modos de establecimiento de políticas lingüísticas: a) a través del plano jurídico legal (leyes, decretos, reglamentos); b) la consuetudine de los actos y prácticas lingüísticas y las orientaciones valorativas sobre ellas, y c) en el marco de las políticas educativas (fundamentalmente en forma de leyes de educación y programas curriculares).

Un análisis retrospectivo de las disposiciones existentes en materia de políticas lingüísticas nos permite afirmar que éstas son prácticamente inexistentes en lo que refiere al primer modo de establecimiento. Es decir, el Uruguay, así como muchos otros países, ha optado por no incluir disposiciones de políticas lingüísticas dentro de su ordenamiento jurídico y de organización administrativa del Estado. La consuetudine expresada en el discurso de la sociedad uruguaya y el ámbito de la educación han sido, en cambio, los espacios de establecimiento explícito o implícito de las políticas lingüísticas en el Uruguay. En particular, el tercer modo de establecimiento, el que vincula el ordenamiento lingüístico al educativo, ha sido el fundamental. Este modo de establecimiento de políticas lingüísticas tiene dos niveles fundamentales: por un lado, las leyes de educación y por otro, los programas curriculares.

Como se explica en detalle en Behares y Brovetto (2009a y 2009b), los actos explícitos de planificación lingüística contenidos en las leyes de educación son muy escasos en la legislación uruguaya desde 1877 (Decreto - Ley de Educación Común, o Ley de Varela) hasta 2008. En el período transcurrido entre 1877 y 1985 (Ley No 15.739 vigente hasta 2008), pueden ser consignados dos actos de planificación lingüística propiamente dichos (Ley Varela, de 1877 y Ley No 14.101, de 1973); ambos hacen referencia de modo indirecto y sin nombrarlo, al español. No se consignan en las leyes, referencias a otras lenguas ${ }^{7}$. Así, es claro que en Uruguay gran parte de las acciones específicas en el establecimiento de las políticas lingüísticas descansa en el sistema educativo y, en particular, en el diseño de programas curriculares, más que en las leyes de educación. En relación a lo anterior, el análisis a través del tiempo de la interacción entre leyes de educación y diseños curriculares permite señalar que han existido entre ellos escasas interacciones, posiblemente debido a sus diversos agentes de origen como consecuencia del carácter autónomo del poder ejecutivo de la educación pública uruguaya y al peso diferencial que el consenso social y la consuetudine tienen sobre estos agentes (Behares y Brovetto, 2009a).

7. Es necesario señalar aquí que las leyes de educación en Uruguay incluyen escasas referencias a contenidos educativos - lingüísticos o de otra índole - y se han enfocado casi exclusivamente en cuestiones relacionadas a los objetivos generales de la educación, y a la administración, gestión y gobierno de la educación (Behares y Brovetto, 2009a).

Pro-Posiçōes, Campinas, v. 21, n. 3 (63), p. 25-43, set./dez. 2010 
Ley General de Educación No. 18.437

Excepcionalmente a esta tradición, la nueva Ley General de Educación (No 18.437), promulgada en 2008, incluye una breve, pero densa en contenidos, referencia a las cuestiones lingüísticas. En esta ley, la "educación lingüística" es una de las nueve líneas transversales a contemplar en el Sistema Nacional de Educación (MEC, 2008: Capítulo VII, Art. 40, Inc.5):

La educación lingüística tendrá como propósito el desarrollo de las competencias comunicativas de las personas, el dominio de la lengua escrita, el respeto de las variedades lingüísticas, la reflexión sobre la lengua, la consideración de las diferentes lenguas maternas existentes en el país (español del Uruguay, portugués del Uruguay, lengua de señas uruguaya) y la formación plurilingüe a través de la enseñanza de segundas lenguas y lenguas extranjeras.

En este párrafo, destinado a la "educación lingüística", la ley reconoce la condición heterogénea en materia lingüística de la sociedad uruguaya y la integra a los propósitos de la educación como dimensión a considerar en lo educativo. Puede interpretarse que se hace referencia a la doble fuente de heterogeneidad que mencionábamos en la sección 2 (variedades del español y de otras lenguas) y se menciona explícitamente al portugués del Uruguay como lengua materna existente en el país. De modo concomitante al reconocimiento de la heterogeneidad lingüística propia, se expresa la intención de educación plurilingüe.

Aunque se puede señalar el carácter ambiguo de la expresión "consideración" (ver Behares y Brovetto, 2009b), pueden hacerse al menos dos anotaciones: por un lado, "consideración" supone que cualquier propuesta educativa que involucre a la frontera debe tomar en cuenta el hecho de que se trata de una sociedad bilingüe; y por otro, es posible interpretar que la ley se abstenga de realizar recomendaciones técnicas específicas que, en el ordenamiento educativo uruguayo, han correspondido a la ANEP. De todas maneras, el mero hecho de consignar en la ley la existencia del portugués del Uruguay como lengua materna de una parte de la población constituye un hecho de notable trascendencia, a la luz del análisis histórico.

\section{Programa de Educación Inicial y Primaria}

El programa de Educación Inicial y Primaria aprobado en 2008 introduce oficialmente la enseñanza de lenguas distintas al español, que quedan así consagradas como componentes del currículo escolar. En concordancia con la propuesta general de políticas lingüísticas de ANEP (ANEP, 2008), se establecen 
el inglés y el portugués como lenguas de enseñanza obligatoria y se define al sistema como plurilingüe:

El presente programa es esencialmente plurilingüe, abriendo la posibilidad de incluir la enseñanza de otras lenguas, además de las ya existentes en el sistema, en función de la demanda social y la presencia de comunidades lingüísticas que lo justifiquen, así como la disponibilidad de recursos humanos y materiales necesarios para su implementación. (CEP, 2008, p. 58).

En lo que tiene que ver con la enseñanza de portugués, el programa reconoce el estatus diverso del portugués para el Uruguay:

En Uruguay la lengua portuguesa es lengua materna o segunda lengua para los escolares de las localidades fronterizas y lengua extranjera para los del resto del país. (CEP, 2008, p. 60).

En lo que refiere específicamente al contexto fronterizo, las motivaciones para el trabajo con portugués se expresan en el siguiente fragmento:

...la motivación para introducir la enseñanza del portugués (lengua materna para algunos niños y segunda lengua para otros) en el contexto fronterizo, deriva en primera instancia de la necesidad de reconocer y respetar la identidad lingüística de la población escolar de esa zona, pero también - y no menos importante - de la localización geográfica de nuestro país, que plantea la necesidad de comunicación a nivel regional. (CEP, 2008, p. 59).

$\mathrm{Al}$ igual que en la Ley General de Educación, se trata de la primera vez que un programa oficial de Educación Primaria con carácter nacional reconoce y atribuye carácter de lengua materna a la variedad vernacular del portugués de frontera. Este reconocimiento constituye de por sí un acto formal fundamental, especialmente teniendo en cuenta la impronta fuertemente monolingüe que ha caracterizado a la escuela pública uruguaya ${ }^{8}$.

La fundamentación de la introducción del portugués incluye también cuestiones referidas a la "localización geográfica" del Uruguay y la "comunicación regional". En otro fragmento se señala incluso la "necesidad de una integración lingüística” en el contexto de la integración económica, social, política y cultural de los países del Cono Sur (CEP, 2008, p. 59). Este argumento queda

8. En Behares y Brovetto (2009a) interpretamos que la Educación Primaria ha estado regida de un modo implícito por el Art. 38 de la Ley Varela citado antes (ver Sección 4), que ha funcionado como estructurador simbólico principal, incluso luego de su derogación (Decreto Ley de Educación Común, Art. 38: "En todas las escuelas públicas la enseñanza se dará en el idioma nacional.")

Pro-Posiçōes, Campinas, v. 21, n. 3 (63), p. 25-43, set./dez. 2010 
instalado como persistencia discursiva (ver nota 6), como parte incorporada al imaginario social uruguayo, aunque sus contenidos o resultados concretos no estén explicitados y probablemente sean muy difusos para la mayor parte de la sociedad uruguaya.

El programa se detiene en el portugués e inglés, en tanto lenguas de enseñanza obligatoria, pero hace explícita la definición como sistema plurilingüe en tanto posibilidad. De modo similar a lo que señalábamos en el análisis de la Ley, el reconocimiento explícito de la condición lingüísticamente heterogénea del Uruguay y de la existencia del portugués como lengua materna, viene unido discursivamente a una intención plurilingüe. Parece constituirse una secuencia discursiva estable entre "Uruguay lingüísticamente heterogéneo" y "educación plurilingüe", donde ambas expresiones se requieren mutuamente, desplazando, al menos parcialmente, el espacio ostentado en leyes y diseños curriculares anteriormente por "el español", como entidad singular, ideal y homogénea.

\section{Puntualizaciones finales}

Este trabajo presenta un panorama y análisis de la educación bilingüe de frontera con especial detenimiento en los programas vigentes y en los marcos legales recientes que introducen actos de políticas lingüísticas.

A modo de conclusión, se plantean a continuación algunas reflexiones y recomendaciones acerca de las necesidades y proyecciones posibles, hacia la estabilización y fortalecimiento de la educación bilingüe de frontera.

Como se presentó en este trabajo, actualmente se desarrollan a nivel de Educación Primaria en área de frontera dos programas que introducen el portugués en diferentes modalidades. En un sentido amplio, y tendiendo en cuenta la centralidad del trabajo con contenidos curriculares, ambos programas pueden considerarse como "educación bilingüe".

Se intentó mostrar aquí que estas propuestas no son ocurrentes innovaciones caprichosas, sino que encuentran sus antecedentes y condiciones de surgimiento en diversas acciones ocurridas dentro y fuera de Primaria, en diversos espacios del sistema educativo, e incluso fuera de la educación, como consecuencia de cambios sociales y culturales de la sociedad fronteriza ocurridos en las últimas décadas. Sin perjuicio de lo anterior, es justo mencionar que los actuales programas de educación bilingüe de frontera, gracias fundamentalmente al trabajo sostenido del equipo técnico, constituyen la primera propuesta que muestra un crecimiento sostenido y una progresiva institucionalización a la Educación Primaria, cuya más clara evidencia es su inclusión en el Programa 2008.

A pesar de este proceso, que se podría considerar de asimilación progresiva de la educación bilingüe fronteriza al sistema educativo, parece necesario señalar 
algunos aspectos que siguen operando como amenazas y sobre los cuales será necesario trabajar para sostener la educación bilingüe de frontera:

a) La educación bilingüe de frontera (en cualquiera de sus modalidades) debe diferenciarse claramente, tanto conceptualmente como en la práctica, de la enseñanza de segundas lenguas y lenguas extranjeras. Especialmente, debe estar muy claro para los distintos actores involucrados la diferencia entre la educación bilingüe de frontera y la enseñanza de portugués en otras regiones no bilingües del Uruguay. En rigor, y por las razones presentadas en extenso en este y otros trabajos, la educación bilingüe español - portugués en área de frontera no es un programa de "enseñanza de lenguas", sino de "inclusión del portugués" en la educación.

b) Las razones y motivaciones para la introducción del portugués en la educación fronteriza deben distinguirse de otro tipo de justificaciones vinculadas a la integración regional y los compromisos asumidos en el marco del MERCOSUR Educativo (ver nota 6). Sin perjuicio de su importancia, estas últimas se refieren al portugués como lengua oficial de Brasil y en ese sentido, se oponen a la justificación para la educación bilingüe de frontera, cuyo centro definitorio es la inclusión del portugués en tanto lengua del Uruguay.

c) En relación a lo anterior, deben distinguirse claramente los programas de educación bilingüe desarrollados en el marco del CEP, de otros posibles programas de intercambio o cooperación, como el "Proyecto de Escuelas Interculturales Bilingües de Frontera", desarrollados en el marco del MERCOSUR Educativo (ver Actas e Informes de la III Reunión Técnica del Proyecto de Escuelas de Frontera). Si bien este tipo de proyectos pueden tener interés y resultados positivos a nivel del intercambio social y cultural entre comunidades vecinas, no constituyen programas bilingües en el sentido desarrollado en este trabajo y tienen potenciales inconvenientes. En particular, el trabajo en portugués con un docente brasileño en una escuela uruguaya trae implícita la idea de que el portugués es la lengua de Brasil, lo cual es contradictorio con los fundamentos de los programas propios.

d) Es necesario el trabajo con el español como segunda lengua en la educación fronteriza. Esta necesidad está mencionada en diversos documentos, pero no ha sido implementada hasta el momento.

En lo que tiene que ver con los nuevos actos de políticas lingüísticos introducidos en 2008, este trabajo muestra que la nueva Ley General de Educación y el nuevo Programa de Educación Inicial y Primaria constituyen eventos novedosos para el Uruguay en materia de políticas lingüísticas, en tanto rompen con una tradición de monolingüismo y omisión del portugués en la educación pública de frontera. De acuerdo al análisis presentado, es posible afirmar lo siguiente: 
a) ambos documentos incorporan de modo concomitante el reconocimiento de la heterogeneidad lingüística del Uruguay y la intención de educación plurilingüe, $y$

b) a diferencia de lo que señaláramos para anteriores leyes y diseños curriculares, donde no era posible registrar relación entre ambos dominios, los actuales documentos muestran consistencia en lo que tiene que ver con el reconocimiento del portugués del Uruguay como lengua materna de parte de la población fronteriza y la necesidad de su consideración en la educación. Es decir, la Ley General de Educación y el Programa de Educación Inicial y Primaria, ambos aprobados en 2008 y con procesos de elaboración parcialmente simultáneos, exhiben en este punto particular una forma de interacción entre el elemento jurídico legal y el de tipo curricular no consignada anteriormente. A modo de cierre, puede considerarse la hipótesis de que esa interacción fue habilitada por un tercer proceso que ofició de agente articulador entre ambos dominios: el Documento de la Comisión de Políticas Lingüísticas para la Educación Pública (ANEP, 2008).

\section{Referencias bibliográficas}

BARRIOS, G. Planificación lingüística e integración regional: el Uruguay y la zona de frontera. In:TRINDADE, A. M.; BEHARES, L. E. (Org.) Fronteiras, educação, integração. Santa Maria: Pallotti, 1996. p. 83-100.

BARRIOS, G. Diversidad ma non troppo: repertorio lingüístico fronterizo y discursos sobre la lengua. In: BARRIOS, G.; BEHARES, L. E. (Org.) Políticas e identidades lingüísticas en el cono sur. Montevideo: Universidad de la República, Facultad de Humanidades y Ciencias de la Educación, Asociación de Universidades Grupo Montevideo, 2006. p. 21-30.

BARRIOS, G.; BEHARES, L.; ELIZAINCÍN, E. et al. Planificación y políticas lingüísticas en Uruguay. Iztapalapa, México, n. 29, p. 76-190, 1993.

BEHARES, L. Diglosia escolar en la frontera uruguaya con Brasil. Matriz social del bilingüismo. Cadernos de Lingüistica Aplicada, Campinas, n.6, p. 228-234, 1984.

BEHARES, L. Planificación lingüistica y educación en la frontera uruguaya con Brasil. Montevideo: Instituto Interamericano del Niño, 1985.

BEHARES, L. E. Portugués del Uruguay y educación fronteriza. In: BROVETTO, C; GEYMONAT, J.; BRIAN, N. (Comp.) Portugués del Uruguay y educación bilingüe. Montevideo: ANEP - CEP, 2007. p. 99-171.

BEHARES, L. E.; ANOLLÉS, P. Evaluación de la primera etapa del Propeler. Rivera: Instituto de Psicología, 1990.

BEHARES, L. E.; BROVETTO, C. Políticas lingüísticas en el Uruguay. Análisis de sus modos de establecimiento. In: BROVETTO, C. (Comp.) Primer Foro Nacional de Lenguas de ANEP. Montevideo: ANEP-CODICEN, 2009a. p. 143-174. 
BEHARES, L. E.; BROVETTO, C. Sobre las referencias al dominio lingüístico en las leyes de educación de Uruguay. In: ENCONTRO INTERNACIONAL DE PESQUISADORES DE POLÍTICAS LINGUÍSTICAS, 4., 2009, Santa Maria: Associação de Universidades Grupo Montevideo/Universidade Federal de Santa Maria, 2009b. p. 95-102.

BERTOLOTTI, V.; CAVIGLIA, S; COLL, M.; FERNÁNDEZ, M. Documentos para la historia del portugués en el Uruguay. Montevideo: Facultad de Humanidades y Ciencias de la Educación. Universidad de la República, 2005.

BORTOLINI, L. S. Letramento em uma escola de educação bilíngüe na fronteira Uruguai/Brasil. 2009. Dissertação (Mestrado) — Universidade Federal do Rio Grande do Sul, Porto Alegre.

BROVETTO, C.; BRIAN, N.; DÍAZ, G.; GEYMONAT, J. Educación bilingüe por inmersión en escuelas públicas del Uruguay. In: ESSARP (Comp.) BilingLatAm 2004. SIMPOSIO INTERNACIONAL SOBRE BILINGÜISMO Y EDUCACIÓN BILINGÜE EN AMÉRICA LATINA, 1. Buenos Aires. Actas... Buenos Aires, 2004. p. 50-61.

BROVETTO, C.; GEYMONAT, J.; BRIAN, N. Una experiencia de educación bilingüe español - portugués en escuelas de la zona fronteriza. IN: BROVETTO, C.; GEYMONAT, J.; BRIAN, N. (Comp.) Portugués del Uruguay y educación bilingüe. Montevideo: ANEP - CEP, 2007. p. 9-47.

CARVALHO, A. M. Rumo a uma definição do português uruguaio. Revista Internacional de Lingüistica Iberoamericana, RILI, v. 1, n. 2, p. 125-149. 2003a.

CARVALHO, A. M. The sociolinguistic distribution of [lh] in Uruguayan Portuguese: A case of dialect diffusion. In: MONTRUL, S.; ORDÓNEZ, F. Linguistic theory and language development in Hispanic languages. Summerville, MA: Cascadilla Press, 2003b. p. 30-44.

CARVALHO, A. M. I speak like the guys on TV: palatalization and the urbanization of Uruguayan - Portuguese. Language Variation and Change. Cambridge University Press, n. 16, p. 127-151, 2004.

CARVALHO, A. M. Diagnóstico sociolingüístico de comunidades escolares fronterizas en el norte del Uruguay. In: BROVETTO, C.; GEYMONAT, J; BRIAN, N. (Comp.) Portugués del Uruguay y educación bilingüe. Montevideo: ANEP - CEP. 2007, p. 49-98.

COLL, M. La frontera heterogénea. Comunicación personal no publicada. 2009.

COLOMBO, S. La formación de maestros y la cuestión de la(s) frontera(s). Aspectos institucionales. In: TRINDADE, A. M.; BEHARES, L. E. (Org.). Fronteiras, educação, integração. Santa Maria: ANEP - CEP, 1996. p. 231-244.

ELIZAINCÍN, A. Algunas precisiones sobre los dialectos portugueses del Uruguay. Montevideo: Dirección General de Extensión Universitaria. Universidad de la República, 1979.

ELIZAINCÍN, A. El español actual en el Uruguay. In: HERNÁNDEZALONSO, C. (Coord.) Historia y presente del español de América. Valladolid: Junta de Castilla y León, 1992. p. 743-758.

ELIZAINCÍN, A. Proyecto Atlas Lingüístico del Uruguay. In: CASTELLO, I. R. et al. (Org.). Práticas de integração nas fronteiras. Temas para o Mercosul. Porto Alegre: UFRGS; Instituto Goethe/ICBA, 1995. p. 217-223. 
GARIBALDI, L.; SALVO, E. Libros de lenguaje 10, $2^{\circ}, 3^{\circ}$ y $4^{o}$ y Guias para el maestro. Montevideo: MECAEP/ANEP/BIRF, 1999a.

GARIBALDI, L.; SALVO, E. Libros de lenguaje $5^{\circ}$ y $6^{\circ}$ y Guias para el maestro. Montevideo: MECAEP/ANEP/BIRF, 1999b.

LORENZO, E. G. E. de. Problemas de la enseñanza del lenguaje en las escuelas fronterizas con el Brasil. Repartido 738 del Consejo Nacional de Enseñanza Primaria y Normal. Montevideo, 1967. Mimeo.

LORENZO, E. G. E. de. Frontier dialect: a challenge to education. The Reading Teacher, v. 28, n. 7, p. 653-658, 1975.

QUESADA, E.; GEYMONAT, J. Elaboração de unidades temáticas para o ensino de português em contexto bilíngüe. In: CONGRESSO NACIONAL DE PROFESSORES DE PORTUGUÊS, 5., 2007. Concordia, Entre Ríos — Argentina. Pôster, 2007.

QUESADA, E.; GEYMONAT, J. O português no ensino fundamental público em contexto fronteiriço. In: CONGRESSO INTERNACIONAL DA SIPLE, 7., 2008, Buenos AiresArgentina, 2008.

RONA, J. P. El dialecto "fronterizo" del norte del Uruguay. Montevideo: Universidad de la República, Facultad de Humanidades y Ciencias, Publicaciones del Departamento de Lingüística. 1959.

URUGUAY. Instrucción Pública. Su Reglamentación Administrativa, del 24 de Agosto de 1877. In: ALONSO CRIADO, Matías. Colección Legislativa de la República Oriental del Uruguay. Montevideo: Imprenta Rural, p. 632-646. [1877], 1978. tomo 4.

URUGUAY. ANEP. Documentos de la Comisión de Politicas Lingüisticas en la Educación Pública. Administración Nacional de Educación Pública. Montevideo: Consejo Directivo Central, 2008a.

URUGUAY. ANEP - CEP Programa de Educación Inicialy Primaria. Administración Nacional de Educación Pública. Montevideo: Consejo Directivo Central. Consejo de Educación Primaria, 2008 b.

URUGUAY. MEC. Ley General de Educación. Ley No. 18.437. Montevideo: Ministerio de Educación y Cultura. República Oriental del Uruguay. Dirección Nacional de Impresiones y Publicaciones Oficiales, 2008c.

Recebido em 04 de fevereiro de 2010 e aprovado em 07 de julho de 2010. 